\title{
Interacción entre poblaciones de Triatoma infestans y Triatoma sordida*
}

\author{
Population interactions between Triatoma infestans and Triatoma sordida
}

\author{
Maria Esther Bar**, Elena Beatriz Oscherov**, Miryam Pieri Damborsky**, Eduardo A. Porcel***, \\ Benedicta Mabel Alvarez**
}

\begin{abstract}
BAR, M.E. et al. Interacción entre poblaciones de Triatoma infestans y Triatoma sordida. Rev. Saúde Pública, 28:59-68, 1994. Para conocer si se producía algún tipo de interacción entre poblaciones de Triatoma infestans y Triatoma sordida que convivian en una unidad experimental y explotaban el mismo recurso alimentario (ave) se las estudió desde setiembre/1988 a abril/1989. La composición etaria inicial para cada especie fue: $27 \mathrm{N1}, 7 \mathrm{~N} 2,11 \mathrm{~N} 3,3 \mathrm{~N} 4,8 \mathrm{N5}, 4$ machos y 10 hembras. La dinámica de población, el estado nutricional, la predación y la conducta gregaria, utilizados como parámetros de comparación, fueron estimados mediante censos mensuales. Triatoma infestans logró mayor éxito colonizador que $T$. sordida dado los valores alcanzados en: fecundidad (146 huevos/hembra), longevidad (157,8 dias) y mortalidad $(39,4 \%)$, en comparación a los obtenidos por $T$. sordida: 118 huevos/hembra, 81,1 días y $54,0 \%$ respectivamente. La población de $T$. infestans tuvo un crecimiento de tipo exponencial, con una alta tasa de renovación ninfal, en contraposición, la población de $T$. sordida se extinguió tempranamente. En general, el peso promedio de los ejemplares de $T$. infestans se mantuvo en valores próximos al inicial, mientras que en $T$. sordida se redujo. Los grupos gregarios se conformaron mayoritariamente en el sector inferior de la pared 1 (próximo al hospedador), observándose mayor nivel de contagio en $T$. infestans. A partir de los resultados obtenidos se formula la hipótesis de la superioridad competitiva en $T$. infestans.
\end{abstract}

Descriptores: Triatoma. Ecología de vectores.

\section{Introducción}

La relación interespecífica puede adoptar diferentes modalidades, por lo que dos poblaciones pueden o no afectarse mutuamente; si lo hacen, la influencia puede ser beneficiosa o adversa. Cuando una población afecta a la otra en forma adversa al requerir ambas un recurso común que es poco abundante, se produce la competencia ${ }^{13}$.

El uso de un mismo recurso puede llevar al solapamiento de sus nichos respectivos, generando coexistencia o exclusión, según la capacidad de cada especie ${ }^{8}$.

$\mathrm{La}$ competencia interespecifica alude a aque-

\footnotetext{
* Investigación subsidiada por el Consejo Nacional de Investigaciones Cientificas y Técnicas (Resolución $\mathbf{n}^{\mathrm{e}}$ 2305/87-259).

** Cátedra de Artrópodos de la Facultad de Ciencias Exactas y Naturales y Agrimensura de la Universidad Nacional del Nordeste

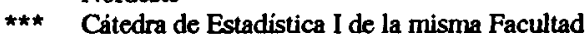

Separatas/Reprints: M.E.Bar - 9 de Julio 1449. 3400. Corrientes, Argentina
}

llas situaciones en las cuales una especie afecta a la población de la otra, por un proceso de interferencia, por ejemplo reduciendo la eficiencia reproductiva o incrementando la mortalidad de su competidor $^{3}$.

Triatoma infestans y Triatoma sordida son las especies de triatominos mejor representadas en la provincia de Corrientes. La primera, principal vector de Trypanosoma cruzi en la Argentina, es esencialmente doméstica, mientras que Triatoma sordi$d a$ se encuentra preferentemente en el peridomicilio. No obstante, ambas especies fueron halladas juntas en la misma vivienda, o en estructuras peridomésticas, pero ocupando sitios diferentes ${ }^{1,16,18}$.

Son numerosos los casos en que el hábitat doméstico ha sido colonizado nuevamente por la misma especie o por otras especies, las que desde sus biótopos invaden al domicilio. Así, por ejemplo, en Brasil se observó que a medida que las poblaciones de $T$. infestans disminuían en las viviendas, éstas eran reinfestadas por $T$. sordida y Panstrongylus megistus ${ }^{16}$.

Otros autores también advierten sobre el peligro de reinfestación ${ }^{4,5,20,21}$. 
En la ciudad de Corrientes, $T$. infestans ha sido sustancialmente eliminada de la vivienda humana principalmente por los programas de control, por lo que se plantea la posibilidad de que $T$. sordida ocupe el nicho vacío dejado por $T$. infestans.

El objetivo del presente trabajo es conocer que tipo de interacción se produce cuando poblaciones de $T$. infestans y $T$. sordida conviven en una unidad experimental y explotan el mismo recurso.

Se ponen a prueba las siguientes hipótesis de trabajo:

- Ambas especies pueden coexistir en la unidad experimental.

- Una especie es dominante, produciéndose la extinción de la otra.

- Una especie es dominante, no produciéndose la extinción de la otra.

\section{Material y Método}

En una unidad experimental (UE) que simulaba un ecótopo doméstico se trabajó con poblaciones de $T$. infestans y $T$. sordida, haciendo notar que no se llevaton a cabo los grupos controles correspon-

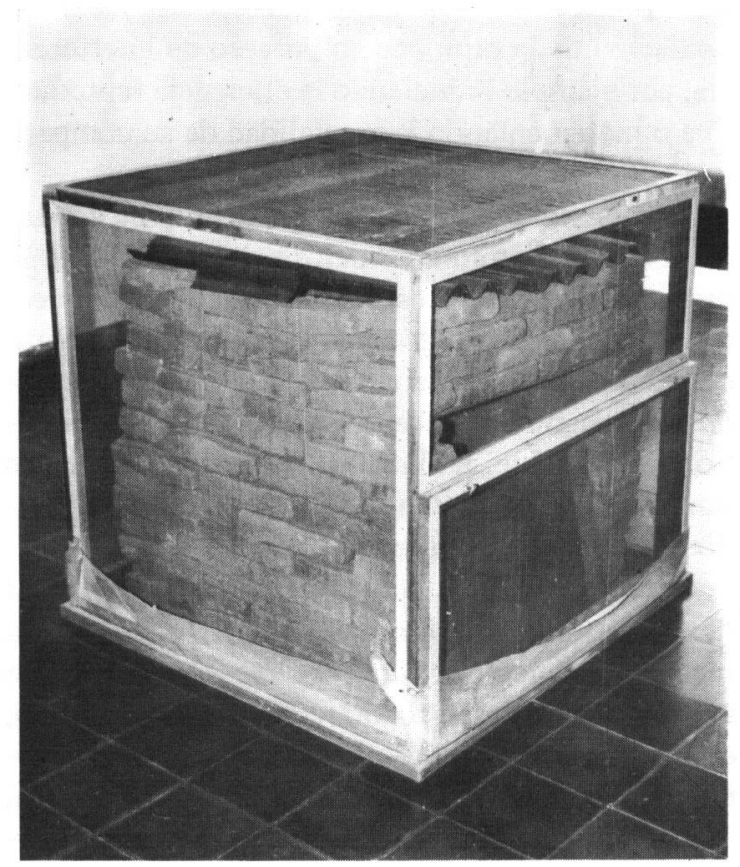

Figura 1. Vista completa de la unidad experimental. dientes a cada una de ellas, por motivos operacionales. La experiencia abarcó ocho meses (setiembre 1988-abril 1989).

La composición etaria inicial para cada especie fue: 27 ninfas de primer estadío (N1), 7 de segundo (N2), 11 de tercero (N3), 3 de cuarto (N4), 8 de quinto (N5), 4 machos y 10 hembras. Los triatominos provenían del insectario de la cátedra de Artrópodos, Facultad de Ciencias Exactas y Naturales (UNNE).

La UE fue construída con paredes de ladrillos de adobe y techo de chapas de cartón y estaba protegida exteriormente por un bastidor de malla plástica y armazón de madera. Sus medidas fueron: $80 \mathrm{~cm}$. de frente, $80 \mathrm{~cm}$. de altura y $80 \mathrm{~cm}$. de profundidad, (Fig. 1 y 2 ) respondiendo a la estructura básica usada por Gorla y Schofield ${ }^{6}$. El recurso alimentario utilizado consistió en un ave (gallina).

Se tomaron como parámetros de comparación: la dinámica de población de cada especie, el estado nutricional de ambas poblaciones (medido a través del peso), la predación y la conducta gregaria, es decir características que permitieran determinar si se producía interacción entre ellas.

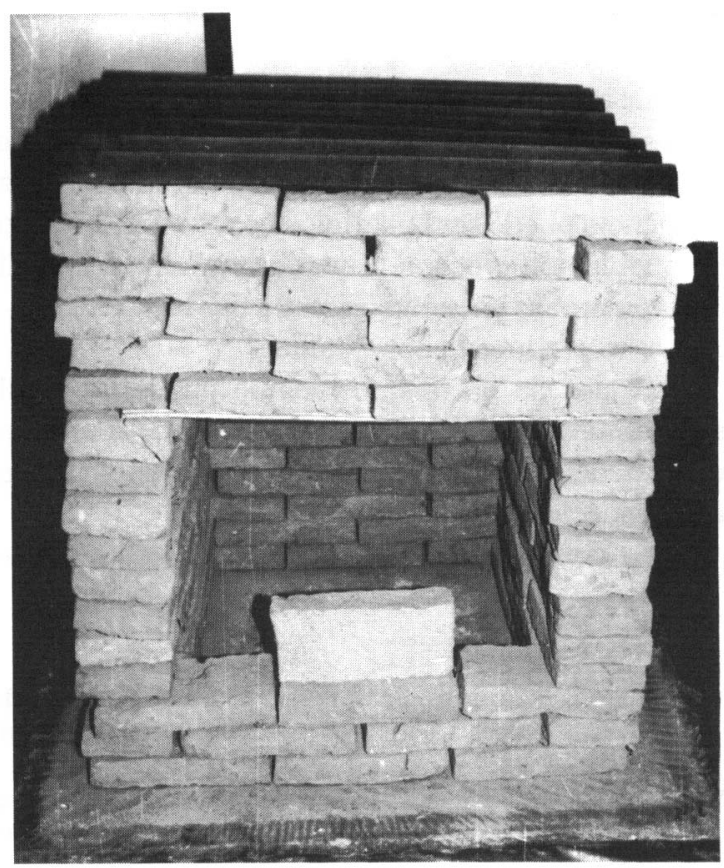

Figura 2. Detalles de la pared frontal y del techo de la unidad experimental. 
Para ello, mensualmente la UE fue desarmada para censar la población de ambas especies. En cada censo se contabilizaba el total de huevos (embrionados, eclosionados y colapsados), ninfas (vivas, muertas y exuvias) e imagos vivos y muertos. Los datos sobre reclutamiento y longevidad de las poblaciones fueron obtenidos mediante el marcado de los ejemplares con pintura acrilica. Las ninfas de primer a quinto estadío fueron marcadas en los segmentos posteriores del abdomen, y los adultos en el pronoto, con una señal de color, según especie y mes.

La fecundidad mensual fue obtenida como la razón entre el total de huevos (embrionados, eclosionados y muertos) y el número de hembras vivas marcadas más el 0,25 de las hembras vivas sin marcar, al tiempo $t^{7}$.

La mortalidad fue calculada como la razón entre el número de muertos de una edad $\mathrm{x}$ y el número de vivos a esa edad $\mathrm{x}$ por 100 .

La predación fue estimada en base al número de ejemplares marcados que no se recuperaban en cada censo y fue expresada como porcentaje de la mortalidad absoluta.

Los individuos vivos, discriminados por clase de edad y especie fueron pesados con una balanza analítica de precisión Sartorius 2842 (sensibilidad $0,1 \mathrm{mg}$.); para la estimación del peso promedio se tomaron como datos iniciales los valores correspondientes al mes de octubre.
Para el estudio de la conducta gregaria fue seleccionada la pared frontal (№ 1), en la cual fueron considerados tres sectores: superior, medio e inferior. Los grupos gregarios hallados en la citada pared fueron separados en cristalizadores, discriminados por sectores de captura, para analizar la composición de las agregaciones, según clase de edad y especie.

Concluído cada censo los individuos vivos eran reintegrados a la UE y los muertos y las exuvias eran conservados en frascos rotulados.

La temperatura y humedad relativa del aire fueron registradas con un termohigrógrafo SIAP THG - 3. La temperatura osciló entre $18^{\circ} \mathrm{C}$ y $40^{\circ} \mathrm{C}$ con una media de $28^{\circ} \mathrm{C}$, y la humedad relativa varió entre $37 \%$ y $100 \%$ con una media de $65 \%$.

El procesamiento de los datos fue realizado utilizando técnicas de estadística descriptiva e inferencial (test de significación de dos proporciones).

\section{Resultados}

\section{Fecundidad y Fertilidad}

Durante los tres primeros meses de experimentación, en que habia hembras de ambas especies, la fecundidad de $T$. infestans fue mayor que la de $T$. sordida y la mayor tasa de fecundidad se observó en ambas especies en noviembre (Tabla). En $T$.

Tabla. Fecundidad y fertilidad de $T$. infestans y $T$. sordida discriminadas según meses de experimentación.

\begin{tabular}{|c|c|c|c|c|}
\hline \multirow{2}{*}{ Meses } & \multicolumn{2}{|c|}{ T. infestans } & \multicolumn{2}{|c|}{ T. sordida } \\
\hline & $\begin{array}{c}\text { Fecundidad } \\
\text { (Huevos/Hembra) }\end{array}$ & $\begin{array}{c}\text { Fertilidad } \\
\%\end{array}$ & $\begin{array}{c}\text { Fecundidad } \\
\text { (Huevos/Hembra) }\end{array}$ & $\begin{array}{c}\text { Fertilidad } \\
\%\end{array}$ \\
\hline Octubre & 85 & 98,41 & 79 & 98,21 \\
\hline Noviembre & 379 & 92,20 & 223 & 99,70 \\
\hline Diciembre & 95 & 81,25 & 79 & 84,88 \\
\hline Enero & 93 & 98,58 & - & - \\
\hline Febrero & 43 & 98,42 & - & - \\
\hline Marzo & 51 & 97,99 & - & 一 \\
\hline Abril & 66 & 96,77 & - & - \\
\hline $\begin{array}{l}\text { Promedio } \\
3 \text { primeros } \\
\text { meses }\end{array}$ & 146 & 93,00 & 118 & 96,90 \\
\hline $\begin{array}{l}\text { Promedio } \\
\text { total }\end{array}$ & 72 & 97,40 & - & - \\
\hline
\end{tabular}


infestans se verificó una correlación negativa ( $\mathrm{r}=$ $-0,54$ ) entre el número de hembras y el promedio de huevos por hembra. La fertilidad en los tres primeros meses de experiencia fue alta siendo mayor en T. sordida $(\mathrm{p}<0,001)$ manteniéndose sin modificaciones en $T$. infestans hasta el final de la experimentación.

Con respecto a la ovipostura, en ambas especies, el primer pico fue observado en noviembre, correspondiéndole a $T$. sordida su mayor valor (668 huevos). El número de huevos disminuyó en diciembre y enero, y en la población de $T$. sordida no hubo más hembras, por lo tanto no se registró más oviposición. En cuanto a $T$. infestans, desde febrero incrementó la puesta hasta alcanzar en abril el mayor número de huevos (1778).

\section{Dinámica ninfal}

La evolución mensual de las N1, N2 y N3 de ambas especies se presenta en la Fig. 3. En $T$. infestans el mayor número de $\mathrm{N} 1$ y N2 se verificó

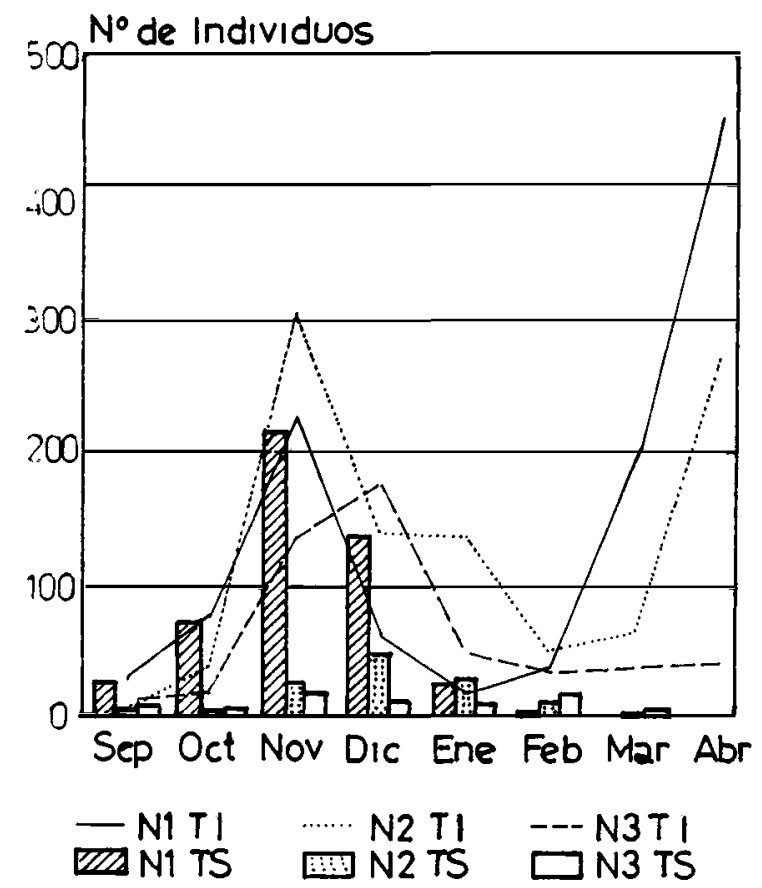

Figura 3. Variación mensual del número de N1-N3 en las poblaciones de $T$. infestans y $T$. sordida. en noviembre y abril. En $T$. sordida las N1 fueron más numerosas en noviembre y diciembre y las $\mathrm{N} 2$ aumentaron 7 veces su número original en diciembre. Las N3 de $T$. infestans fueron aumentando su número hasta diciembre mientras que las de $T$. sordida siempre fluctuaron alrededor de su composición inicial. Las $\mathrm{N} 4$ de T. infestans estuvieron mejor representadas en diciembre y las N5 en enero, valores que si bien decrecieron, se mantuvieron en número superior al inicial, mientras que el número de N4 y N5 de $T$. sordida no varió sustancialmente respecto a sus composiciones originales en ningún momento (Fig. 4).

\section{Dinámica de adultos}

No se registraron aumentos en el número de machos y hembras durante los primeros meses en ninguna de las 2 especies (Fig. 5). En $T$. infestans se evidenció desde febrero, un notable incremento de la población, a diferencia de $T$. sordida cuyos adultos se extinguieron.

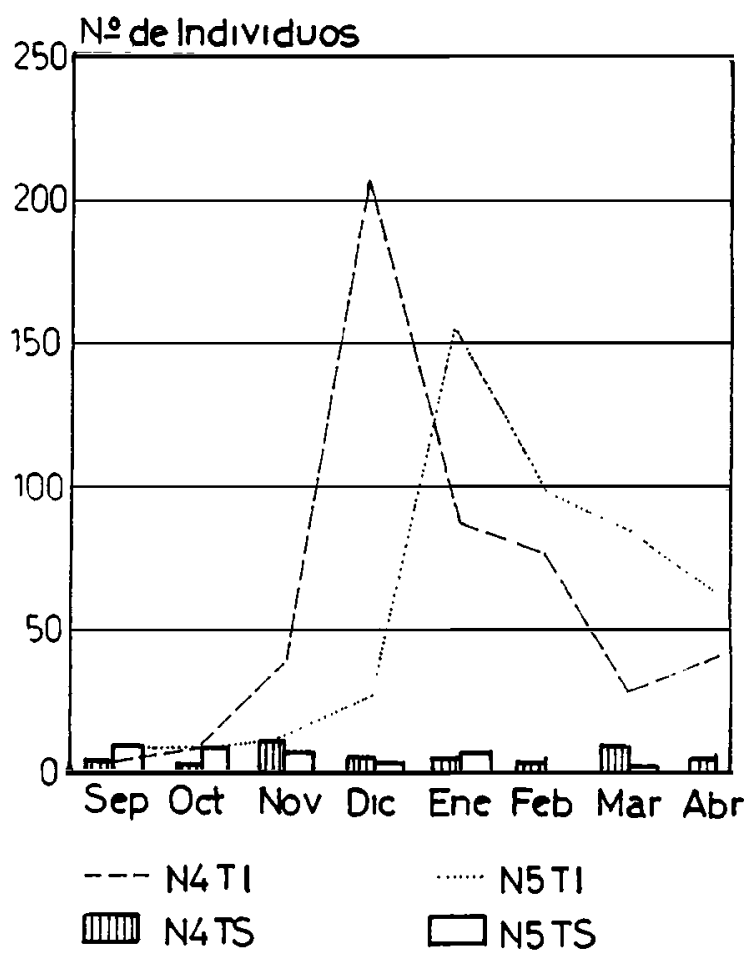

Figura 4. Variación mensual del número de N4 y N5 en las poblaciones de $T$. infestans y $T$. sordida. 


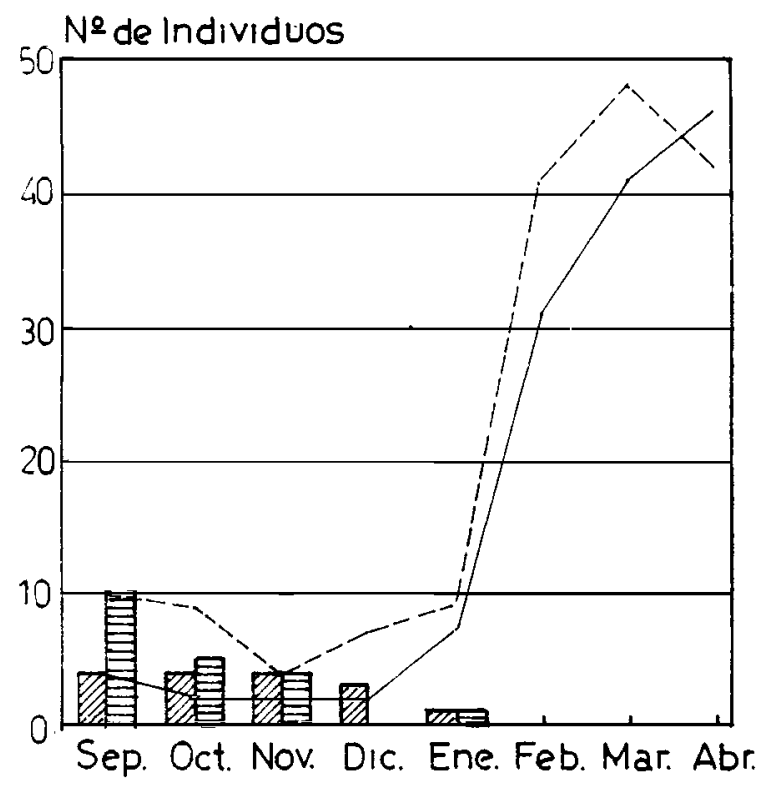

\section{- MachosTI -.- HembrastI \\ Machos TS HembrastS}

Figura 5. Variación mensual del número de hembras y machos de $T$. infestans y $T$. sordida.

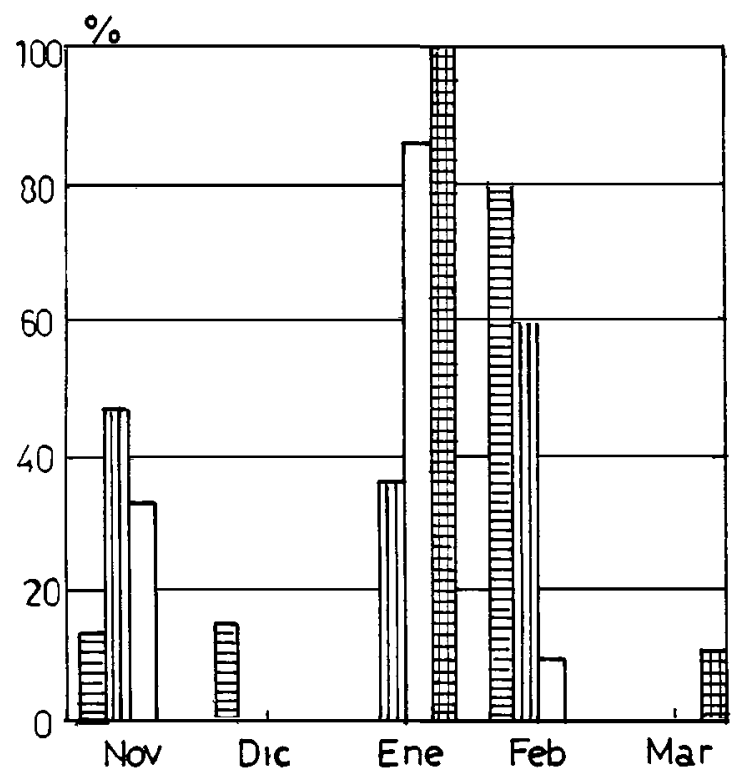

巨N1 皿N2 $\square$ N3 $N$ N4

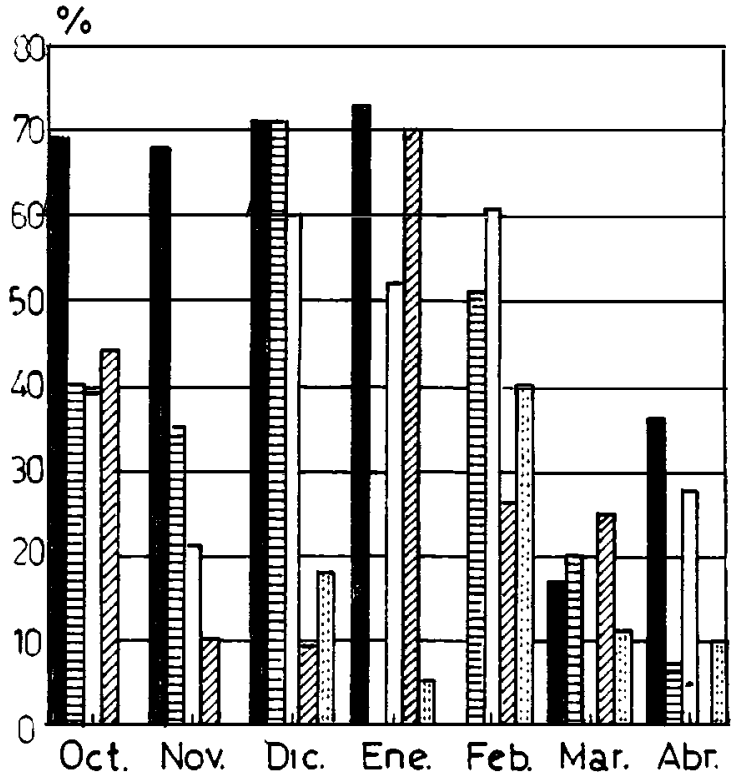

N1

Figura 6. Porcentaje de renovación ninfal mensual de $T$. infestans discriminado por estadios.

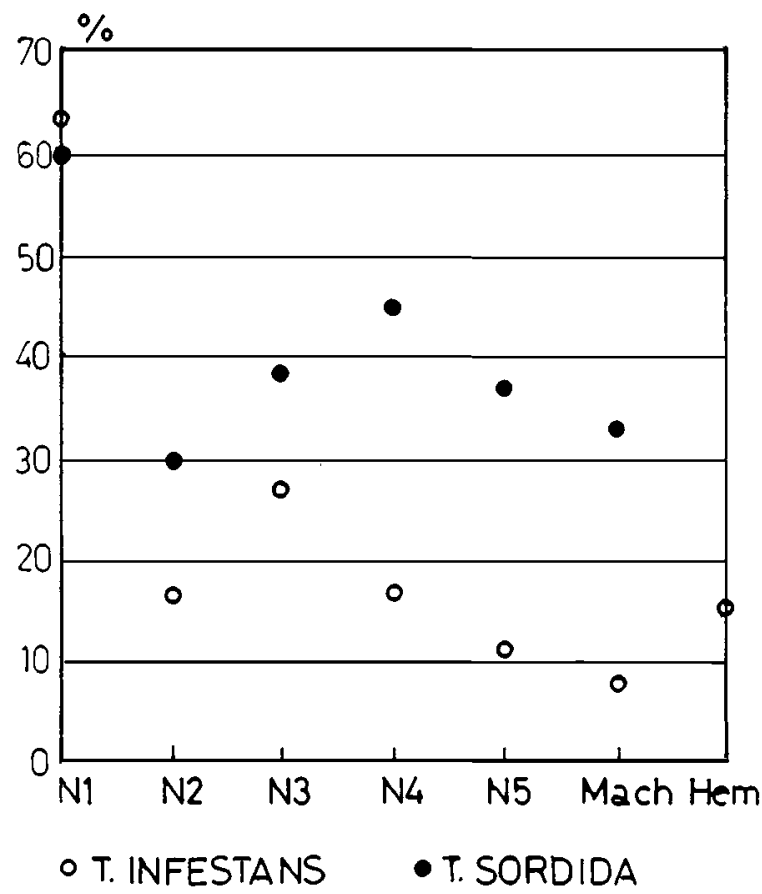

Figura 8. Mortalidad media de $T$. infestans y $T$. sordida según clase de edad. 


\section{Renovación ninfal}

La tasa de renovación de $T$. infestans fue superior a la de $T$. sordida, con valores que en su mayoría, oscilaron entre un 40 y $74 \%$ (Fig. 6). Esta tendencia no se mantuvo ya que desde marzo se registró una disminución en el porcentaje de mudas ( $7-36 \%)$. En general, en $T$. sordida se observaron valores muy variables $(0 \%-100 \%)$ (Fig. 7). La proporción de $\mathrm{N} 3$ y N4 que mudaron al estadio siguiente fue mayor en enero y la de N1 y N2 en febrero, en tanto que en N5 no se constató renovación.

\section{Longevidad}

La longevidad media de adultos de T. infestans fue de 157,8 dias (mín: 63, máx: 304) y la de $T$. sordida de 81,1 días (mín: 35, máx: 131).

\section{Mortalidad}

La mortalidad media para $T$. infestans fue de $39,4 \%$ y $54,0 \%$ para $T$. sordida.

En ambas especies el estadío que presentó mayor mortalidad fue $\mathrm{N} 1(63,4 \%$ para $T$. infestans y $59,8 \%$ para $T$. sordida), valor que decrece bruscamente en el segundo estadio; en el resto de los estadios ninfales y adultos la mortalidad fue notoriamente superior en $T$. sordida, correspondiéndoles a las hembras el mayor porcentaje (Fig. 8).

Asimismo, la mortalidad atribuída a la predación alcanzó valores superiores en $T$. sordida. El 93\% y $94 \%$ de la mortalidad de N1 de $T$. infestans y $T$. sordida respectivamente fue debida a esta causa, mientras que en el resto de las clases de edad la predación media fue menor en $T$. infestans $(24,2 \%)$ que en $T$. sordida $(51,6 \%)$.

\section{Agregación}

Desde noviembre a febrero $T$. infestans y $T$. sordida se agregaron interespecíficamente. El número de ejemplares agregados por especie, de todas las agregaciones registradas a lo largo de la experiencia, se visualiza en la Figura 9. En ambas especies los porcentajes de agregación se mantuvieron en niveles relativamente similares durante los tres primeros meses, con tendencia a disminuir. En fe- brero si bien los porcentajes se incrementaron no alcanzaron los valores de noviembre.

A partir de marzo, aunque en menor número, $T$. infestans continuó agregándose intraespecíficamente ya que $T$. sordida se había extinguido. No obstante, los porcentajes promedios de agregación en ambas especies difirieron significativamente, siendo mayor en $T$. infestans: $31,7 \%$ que en $T$. sordida: $26,7 \%(\mathrm{z}=2,2 \mathrm{p}=0,014)$. Se destaca que en las dos especies el mayor nivel de contagio se observó en N2 y N3 (33,3\% y $38 \%$ respectivamente) siguiéndole en orden de importancia N1.

Los grupos gregarios conformados en la pared 1 , mostraron una marcada preferencia por el sector inferior, concentrando el mismo el $95 \%$ y $99 \%$ de los ejemplares, de $T$. infestans y $T$. sordida respectivamente, comportamiento que se mantuvo a través de los meses de experiencia. T. infestans además formó grupos gregarios intraespecíficos en los sectores medio y superior.

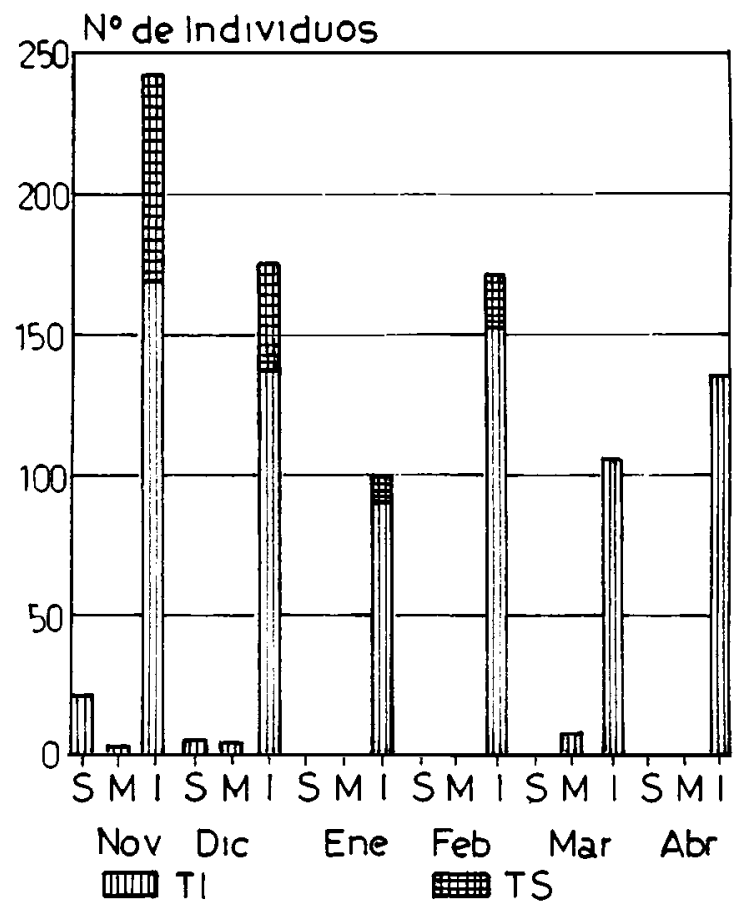

S:superior $M:$ medio $1:$ inferior

Figura 9. Total de individuos agregados de $T$. infestans y $T$. sordida según sectores y mes. 
Peso

Los cuatro primeros estadíos ninfales de $T$. infestans mantuvieron su peso inicial durante la experimentación y salvo excepciones lo superaron levemente. A diferencia, las N5 presentaron un gran incremento en noviembre disminuyendo luego en diciembre y enero; a partir de este mes, los valores fueron relativamente estables; los machos y hembras redujeron su peso inicial no alcanzando a superarlo en toda la experiencia (Fig. 10). En T.sordi$d a$ se observó una tendencia general a la disminución de peso respecto al inicial; excepcionalmente as registraron, para los diferentes estadios, pequeños incrementos (Fig. 11).

\section{Discusión y Conclusión}

En la experiencia con $T$. infestans y $T$. sordida, si bien no fueron incluidas las correspondientes unidades controles, por problemas operativos, se

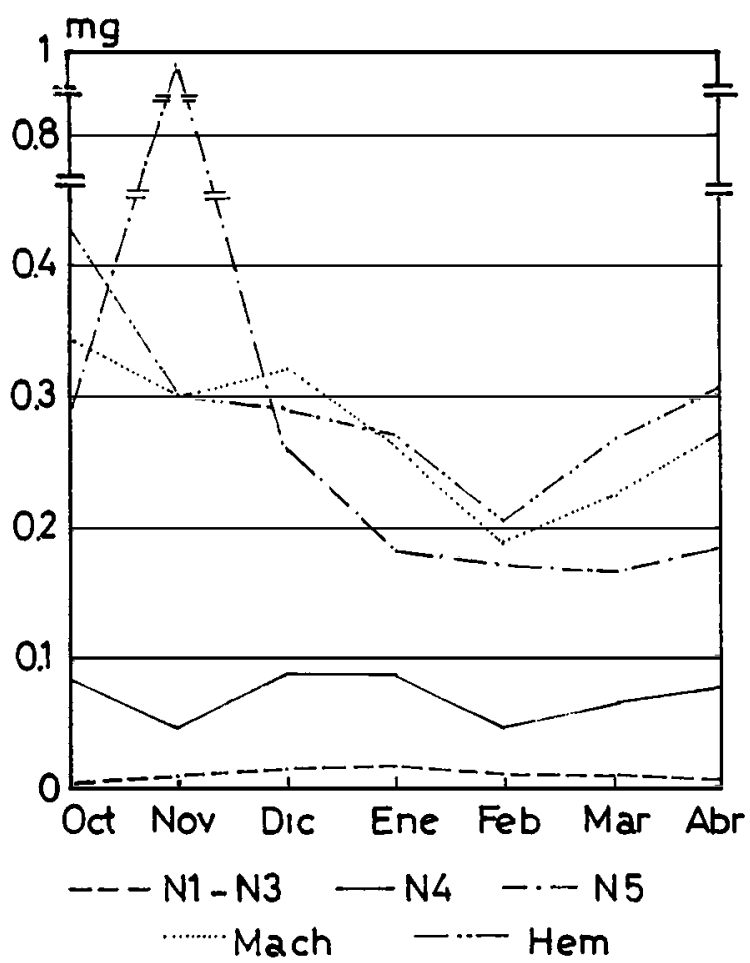

Figura 10. Peso promedio de $T$. infestans diferenciado por clase de edad y mes. obtuvieron resultados interesantes que justifican su análisis, aún teniendo en cuenta el caráter provisorio de los mismos.

En el contexto de lo antes expuesto, se manifiesta que T.infestans tuvo mayor éxito colonizador que $T$. sordida, hecho que se evidencia por las tasas más altas alcanzadas en los diferentes parámetros analizados. En su crecimiento, T. infestans siguió un modelo de tipo exponencial; en contraposición, la población de $T$. sordida llegó a su extinción cuando aún se hallaba en una etapa temprana de crecimiento y expansión.

La fecundidad media de $T$. infestans fue superior a la de $T$. sordida. Las tasas de fecundidad de $T$. infestans y $T$. sordida no se corresponden con el mayor reclutamiento de hembras. La correlación negativa entre el número de hembras y el promedio de huevos por hembra coincide con lo constatado por Gorla y Schofield?

Los porcentajes de fertilidad obtenidos para ambas especies son semejantes a los logrados para

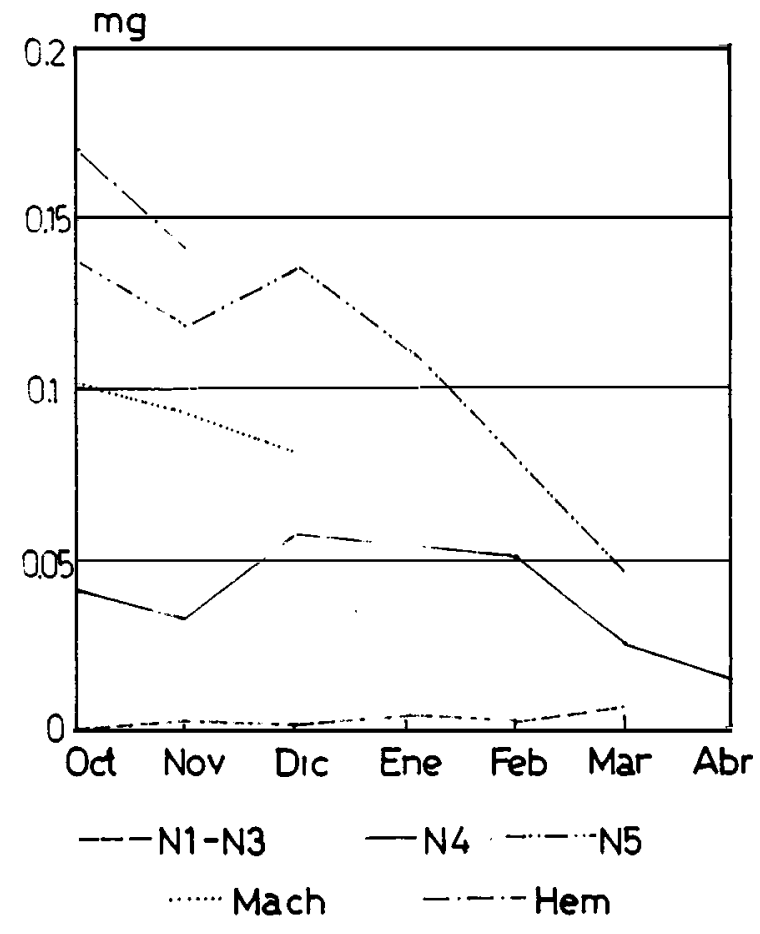

Figura 11. Peso promedio de T. sordida diferenciado por clase de edad y mes. 
T. infestans por Rabinovich ${ }^{14}$ y PerlowagoraSzumlewicz ${ }^{12}$ y para T. sordida por Mello ${ }^{11}$ y Juarez y Castro Silva?.

En T. infestans se observó un pico de emergencia de adultos desde febrero a abril, precedido de valores máximos en la producción de ninfas de tercero y cuarto estadio en diciembre y de quinto en enero, en concordancia con lo verificado para la misma especie, en casas rurales de Mambaí, Bra$\mathrm{sil}^{18}$.

Si bien la renovación ninfal fue más elevada en T. infestans, los patrones generales de reclutamiento de ambas especies, presentaron grandes variaciones entre los diferentes estadíos.

La renovación ninfal de las especies en estudio, no mostró relación estrecha con la temperatura y humedad relativa de cría, a diferencia de lo comprobado para $T$. infestans, por Gorla y Schofield ${ }^{7}$.

La longevidad media de $T$. infestans fue el doble de la de $T$. sordida y semejante a la obtenida por Rabinovich ${ }^{14}$.

La mortalidad de ambas especies, discriminada por estadios presenta patrones de comportamiento similares, aunque en $T$. sordida los valores fueron ostensiblemente superiores.

$\mathrm{Si}$ bien $T$. sordida cumple óptimamente su ciclo de vida tanto en ecótopos peridomiciliarios como en condiciones de laboratorio, en esta experiencia en la cual compartió el hábitat con otra especie, respondió de manera diferente. Este comportamiento, tal vez, podría explicarse en el hecho de que miembros de una misma especie que están protegidos de su predator en una zona concreta, pueden ser extremadamente vulnerables al mismo predador, en un hábitat distinto ${ }^{13}$.

Por su parte, $T$. infestans demostró habilidad para minimizar la mortalidad asociada al hospedador, dado que los porcentajes de predación fueron apreciablemente menores que los verificados en todos los estados de desarrollo de $T$. sordida, excepto en el primer estadio ninfal. Cabe destacar, además, que se verificó una notoria diferencia en la proporción de hembras predadas de $T$. sordida, con un valor 4,6 veces mayor al de $T$. infestans. También es importante señalar que la mortalidad elevada, incluída la predación y la falta de reclutamiento de imagos de $T$. sordida negativizaron el efecto que podría haber tenido la alta fertilidad, en el incremento de la población.
La conformación de los grupos gregarios interespecificos en el sector inferior de la pared 1, permiten confirmar la importancia de la cercanía del recurso alimenticio, en coincidencia con lo constatado por Ronderos et al. ${ }^{17}$ en una población doméstica de $T$. infestans.

Esta conducta de selección del refugio contribuiría a minimizar los riesgos para la obtención de alimento, disminuyendo la distancia a recorrer, tal como lo señala Rabinovich ${ }^{15}$. Del análisis de la composición cuantitativa de las agregaciones surge que el nivel de contagio fue más marcado en $T$. infestans. El predominio de estadíos ninfales sobre adultos, está en el orden de los resultados hallados para $T$. phyllosoma y Rhodnius prolixus por Rabinovich ${ }^{15}$.

En general, el peso promedio de los ejemplares de $T$. infestans se mantuvo en valores próximos al inicial, mientras que en $T$. sordida se redujo, hecho que podria ser atribuído a factores tales como la interferencia producida entre ambas, $o$ a que el aumento de la población de $T$. infestans habría actuado en desmedro de $T$. sordida, principalmente en los últimos meses de la experiencia. Acerca de ello, Schofield ${ }^{18,19}$ y Rabinovich ${ }^{15}$ señalan que a mayores densidades corresponde un estado nutricional más bajo.

A partir de los resultados obtenidos en esta primera parte del trabajo sobre la interacción entre ambas especies se formula la hipótesis de la superioridad competitiva de $T$. infestans.

Crombie $^{2}$ manifiesta que una de las formas de expresar la superioridad de una especie respecto a otra está dada por: la tasa de reproducción y superviviencia combinadas; al respecto $T$. infestans mostró valores superiores en los parámetros antes señalados.

Pianka ${ }^{13}$ expresa que cuando dos especies entran en competencia y el sistema avanza hasta la saturación, una de las especies elimina por completo a la otra, produciéndose una exclusión competitiva, en esta experiencia la tasa real de incremento de $T$. sordida declinó a valores negativos, mientras que la de $T$. infestans continuaba creciendo.

La hipótesis anteriormente planteada podrá ser verificada al replicarse la experiencia, incluyendo además una unidad experimental control con $T$. infestans y otra con $T$. sordida. Cabe resaltar que recién entonces podrá confirmarse si la extinción de 
T. sordida se debió a la superioridad competitiva de $T$. infestans o a que $T$. sordida no fue capaz de sobrevivir en el sistema experimental empleado.

\section{Agradecimientos}

Al Dr. David E. Gorla por la lectura crítica del manuscrito; a la Prof. Ma E. Varela por la colaboración en la disección mensual del ecótopo artificial; a la Arq. Susana Pouppard por el diseño de los gráficos.

BAR, M.E. et al. [Population interactions between Triatoma infestans and Triatoma sordida]. Rev. Saúde Pública, 28:59-68, 1994. Populations of $T$. infestans and T. sordida were studied between September 1988 and April 1989 with a view to discovering if any kind of interaction took place between them while they lives together in the same experimental unit and exploited the same food resource (chicken). The initial age structure for each species was: 27 N1, $7 \mathrm{~N} 2,11 \mathrm{~N} 3,3 \mathrm{~N} 4,8 \mathrm{N5}, 4$ males and 10 females. The population dynamics nutritional status, predation and gregarious behavior were estimated by means of a monthly census. The colonizing success of $T$. infestans was greater than that of $T$. sordida in view of the values obtained: fecundity (146 eggs/female), longevity (157.8 days) and mortality (39.4) compared with the values recorded for $T$. sordida: $118 \mathrm{eggs} / \mathrm{female}, 81.1$ days and $54.0 \%$ respectively. The population growth of $T$. insfestans followed an exponential model, with a high nymphal recruitment rate, while the $T$. sordida population was early extinguished. In general, the average weight of $T$. infestans remained close to the initial values while that of $T$. sordida declined. Gregarious groups were formed principally in the inferior sector of wall 1 (near the host) with a higher aggregation in $T$. infestans. These results make it possible to propose the hypothesis of the competitive superiority of $T$. infestans.

\section{Keywords: Triatoma. Ecology, vectors.}

BAR, M.E. et al. Interação entre população de Triatoma infestans e Triatoma sordida. Rev. Saúde Pública, 28:59-68, 1994. Para saber se algum tipo de interação se produzia entre populações de Triatoma infestans e $T$. sordida que conviviam em uma unidade experimental e compartilharam - mesmo recurso alimentar (ave), foi realizado estudo que abrangeu o periodo de setembro de 1988 a abril de 1989. A composição etária inicial para cada espécie foi: $27 \mathrm{~N} 1,7$ $\mathrm{N} 2,11 \mathrm{~N} 3,3 \mathrm{~N} 4,8 \mathrm{N5}, 4$ machos e 10 fêmeas. A dinâmica da população, o estado nutricional, a predação e a conduta gregária foram estimados mediante censos mensais. $T$. infestans obteve maior êxito colonizador que $T$. sordida dado os valores alcançados em: fecundidade (146 ovos/fêmea), longevidade (157,8 dias) e mortalidade $(39,4 \%)$, em comparação aos obtidos por T. sordida: 118 ovos/fêmeas, 81,1 dias e $54,0 \%$ respectivamente. A população de $T$. infestans teve crescimento do tipo exponencial, com alta taxa de renovação ninfal; em contraposição, a população de $T$. sordida se extinguiu mais cedo. Em geral, o peso médio dos exemplares de $T$. infestans manteve-se em valores próximos ao inicial, enquanto que em $T$. sordida se reduziu. Os grupos gregários se formaram sobretudo no setor inferior da parede 1 (próximo ao hospedeiro), observando-se maior nivel de contágio em $T$. infestans. A partir dos resultados obtidos, formulou-se a hipótese da superioridade competitiva de T. infestans.

Descritores: Triatoma. Ecologia de vetores.

\section{Referencias Bibliográficas}

1. BAR, M.E.; OSCHEROV, E.B.; DAMBORSKY, M.P., VARELA, M.E.; MIZDRAJI, G.; PORCEL, E. Triatomismo del Departamento San Luis del Palmar de la Provincia de Corrientes, Argentina. Medicina (B. Aires), 52: 193-201, 1992.

2. CROMBIE, A.C. Interspecific competition. J.Anim.Ecol, 16: 44-73, 1947.

3. ELTON, C.S. \& MILLER, R.S. The ecological survey of animal communities: with a practical system of classifying habitats by structural characters. J.Ecol., 42: 460-96, 1954.

4. FORATTINI, O.P.; ROCHA SILVA, E.O.; FERREIRA, O.A.; RABELLO, E.X.; PATTOLI, D.G.B. Aspectos ecológicos da tripanossomose americana. III Dispersāo local de triatomíneos, com especial referência ao Triatoma sordida. Rev.Saúde Pública, 5: 193-205, 1971.

5. GAMBOA CUADRADO, J. Factores que determinan la infestación y reinfestación triatominea de la vivienda campesina. Bol.Inf.Dir. Malariol y San.Amb., 13:3-5, 1973.

6. GORLA, D.E. \& SCHOFIELD, C.J. Analysis of egg mortality in experimental populations of Triatoma infestans under natural climatic conditions in Argentina. Bull.Soc. Vector Ecol., 10: 107-17, 1985.

7. GORLA, D.E. \& SCHOFIELD, C.J. Population dynamics of Triatoma infestans under natural climatic conditions in the Argentine Chaco. Med.Vet.Entomol, 3: 179-94, 1989.

8. HUTCHINSON, G.E. Concluding remarks. Cold Spring Harbor Symp. Quant. Biol., 22: 415-27, 1957.

9. JUAREZ, E. \& SILVA, E.P. de C. Comportamento do Triatoma sordida em condições de laboratório. Rev.Saúde Pública, 16 (supl.): 1-36, 1982.

10. MARSDEN, P.D.; ALVARENGA, N.J.; CUBA, C.C.; SHELLEY, A.J.; COSTA, C.H.; BOREHAM, P.F.L. Studies of the domestic ecology of Triatoma infestans by means of house demolition. Rev. Inst. Med. Trop. Säo Paulo, 21: 13-25, 1979.

11. MELLO, D.A. Some aspects of feeding habits under laboratory conditions of Triatoma sordida (Stal), 1859) and Rhodnius neglectus Lent, 1954 (Hemiptera, Reduviidae). Rev.Bras.Biol.,40: 323-6, 1980.

12. PERLOWAGORA-SZUMLEWICZ, A. Estudos sobre biologia do Triatoma infestans, o principal vetor da doença de Chagas no Brasil (importância de algumas de suas características biológicas no planejamento de esquema de combate a esse vetor). Rev.Bras.Malariol.Doenças Trop., 21: $117-60,1969$. 
13. PIANKA, E.R. Ecología evolutiva. Barcelona, Ed. Omega, 1982.

14. RABINOVITCH, J.E. Vital statistics of Triatominae (Hemiptera: Reduviidae) under laboratory conditions. I. Triatoma infestans Klug. J.Med.Entomol., 9: 351-70, 1972.

15. RABINOVITCH, J.E. Ecología poblacional de los triatominos. In: Carcavallo, R.V.; Rabinovitch, J.E.; Tonn, R.J., eds. Factores biológicos y ecológicos de la enfermedad de Chagas. Buenos Aires, Servicio Nacional de Chagas, 1985. v.1, p.121.47.

16. ROCHA e SILVA, E.O. da; DIAS JUNIOR, J.; FERREIRA GUARITA, O. Suspensão do rociado no combate ao Triatoma infestans em áreas do Estado de São Paulo, Brasil. Rev.Saúde Pública, 3: 173-81, 1969.

17. RONDEROS, R.A.; SCHNACK, J.A.; GHILINI, J.M.; SPINELLI, G.R. Estudio ecológico sobre una población domiciliaria de Triatoma infestans Klug de la provincia biogeográfica chaqueña. ECOSUR, 8(15): 1-24, 1981.

18. SCHOFIELD, C.J. Density regulation of domestic populations of Triatoma infestans in Brazil. Trans.R.Soc.Trop.Med. Hyg., 71: 761-69, 1980.

19. SCHOFIELD, C.J. The role of blood intake in density regulation of populations of Triatoma infestans (Klug) (Hemiptera: Reduviidae). Bull.Entomol.Res., 72: 617-29, 1982.

20. TONN, R.J.; ESPINOLA, H.; MORA, E.; JIMENEZ, J.E. Trampa de luz negra como método de captura nocturna de triatominos en Venezuela. Bol.Dir.Malariol.San.Amb., 18: 25-30, 1978.

21. ZELEDON, R. \& RABINOVITCH, J.E. Chagas' disease: an ecological appraisal with special emphasis on its insect vectors. Ann.Rev. Entomol., 26: 101-33, 1981.

Recebido para publicaçāo em 3.3 .1993

Reapresentado em 8.9.1993

Aprovado para publicação em 27.10.1993 